\title{
Comparison of chemiluminescence immunoassay, enzyme-linked immunosorbent assay and passive agglutination for diagnosis of Mycoplasma pneumoniae infection
}

This article was published in the following Dove Press journal:

Therapeutics and Clinical Risk Management

\section{Dongmiao Chen ${ }^{1, *}$ \\ Yajie Zhang ${ }^{1, *}$ \\ Yinjuan $\mathrm{Xu}^{2}$ \\ Tingting Shen' \\ Guorui Cheng' \\ Bingkang Huang' \\ Xiandong Ruan ${ }^{2}$ \\ Congrong Wang'}

'Department of Laboratory Medicine, Nanfang Hospital, Southern Medical University, Guangzhou 5 I05I5,

Guangdong, China; ${ }^{2}$ Department of Laboratory, Xintang Hospital, Southern Medical University,

Zengcheng, Guangzhou 5II340,

Guangdong, China

*These authors contributed equally to this work
Correspondence: Congrong Wang Department of Laboratory, Xintang Hospital, Southern Medical University, Number 10 Shuisong Road, Xintang, Zengcheng, Guangzhou 5 II340,

Guangdong, China

Tel +860208277 7979

Email 137II443959@I63.com

\begin{abstract}
Objective: This study aimed to compare the performance of chemiluminescence immunoassay (CLIA), enzyme-linked immunosorbent assay (ELISA), and passive agglutination (PA) method in detecting Mycoplasma pneumoniae (MP) infection.

Methods: This study enrolled a total of 280 patients who were consecutively seen at the Nanfang Hospital of the Southern Medical University in Guangdong Province, China, between August and December 2016. Serum was collected and examined by CLIA, ELISA, and PA, respectively.

Results: There were 180 positive (64.3\%) and 100 negative cases (35.7\%) by PA, 184 positive (65.7\%) and 96 negative cases (34.3\%) by CLIA MP- immunoglobulin (Ig) M, 89 positive (31.8\%) and 191 negative cases $(68.2 \%)$ by CLIA MP-IgG, 196 positive (70\%) and 84 negative cases (30\%) by ELISA MP-IgM, and 114 positive (40.7\%) and 166 negative cases (59.3\%) by ELISA MP-IgG. Patients were allocated to two groups based on PA results. In PA-negative group ( $\leq 1: 40)$, the positive rates of MP-IgM by CLIA were $22.8 \%$ and $51.2 \%$ and by ELISA were $33.3 \%$ and $53.5 \%$, respectively. In the PA-positive group (1:80 to $\geq 1: 1,280)$, MP-IgM negative cases showed a decreasing trend: $40 \%, 18 \%, 14.3 \%, 10 \%$, and $6.7 \%$ (CLIA), and $43.3 \%, 8 \%, 14.3 \%, 5 \%$, and $6.7 \%$ (ELISA). The consistency between CLIA/ELISA MP-IgM, -IgG, and -IgG+MP-IgM was $>92 \%$ for negative cases and $>75 \%$ for positive cases, resulting in an overall consistency rate $>88 \%$. The kappa coefficients were $0.804,0.763$, and 0.806 , respectively.

Conclusion: CLIA and ELISA have a higher sensitivity compared with PA. CLIA has a high concordance with ELISA. Moreover, CLIA has a higher specificity and sensitivity for the detection of $\operatorname{IgM}$ and $\operatorname{IgG}$ and should be used for the clinical diagnosis of MP infection.
\end{abstract}

Keywords: chemiluminescence immunoassay, enzyme-linked immunosorbent assay, passive agglutination, mycoplasma pneumonia antibody

\section{Introduction}

Mycoplasma pneumoniae (MP), a very small bacterium in the class Mollicutes, is a human pathogen that causes the disease mycoplasma pneumonia. MP exclusively parasitizes the respiratory tract epithelium of humans ${ }^{1}$ and may infect people at all ages, with children being most susceptible. MP has been one of the most important pathogens of community-acquired pneumonia (CAP) in children, accounting for around $10 \%-40 \%$ of pediatric CAP cases. ${ }^{2,3}$ Approximately $18 \%$ of MP-infected children require hospitalization in China. ${ }^{4}$ Currently, there are three diagnosis methods for $\mathrm{MP}$, including culture test, polymerase chain reaction (PCR), and serologic test. Nevertheless, none of these methods has been considered as the golden diagnostic method of 
MP infection. Culture test is an important standard for the detection of MP infection, ${ }^{5}$ but is rarely used as diagnostic tool because of the requirement of special culture media, as well as laboratory conditions and technicians. In case that paired sera are available, MP infection can be firmly confirmed when the MP-specific immunoglobulin (Ig) G titer in paired sera increases by at least fourfold, ${ }^{6-8}$ however, such method cannot be used for early diagnosis. Although PCR is rapid and effective in the detection of $\mathrm{MP}$, the procedure cannot distinguish MP carriers from MP infection because MP is present in $0.1 \%-13.5 \%$ of healthy individuals. ${ }^{9}$ Moreover, MP DNA may exist in the upper respiratory tract for 7 weeks to 7 months since the initial infection, leading to false-positive results in PCR test. Currently, elevated serum MP-IgM level is the main laboratory evidence for clinical diagnosis of MP infection. The enzyme-linked immunosorbent assay (ELISA) is the most common diagnostic method of MP detection because of the low cost and relatively high sensitivity and specificity. However, the manual procedure is time-consuming and labor-intensive, and, therefore, is not suitable for rapid detection. Passive agglutination (PA) is a method that has been widely used in the diagnosis of MP infection, but is largely limited by its inability to discriminate between $\mathrm{IgG}$ and $\operatorname{IgM}$ because of nonspecific reactions. ${ }^{10}$ Chemiluminescence immunoassay (CLIA), an effective combination of immunoreaction and chemiluminescent system, has recently drawn increasing attention in MP diagnosis. Herein, this study aimed to clarify the diagnostic value of CLIA for the diagnosis of MP infection in comparison with PA and ELISA.

\section{Patients and methods \\ Patients}

This study enrolled a total of 280 patients who were consecutively seen at the Nanfang Hospital of Southern Medical University in Guangdong Province, China, between August and December 2016. The patients included 150 males (53.6\%) and 130 females $(46.4 \%)$ with a mean age of 4.5 years (range: 10 months to 46 years). According to the diagnosis criteria in the 7 th edition of Internal Medicine, ${ }^{11}$ all patients had respiratory symptoms, including acute bronchitis, bronchial asthma, upper respiratory tract infection, and acute tonsillitis, and thus were suspected to have MP infection. The gold standard test for MP infection is a fourfold increase in MP-specific IgG when comparing acute phase and convalescent sera collected in an interval of 2-4 weeks. Among these 280 patients, 176 were diagnosed with refractory Mycoplasma pneumoniae pneumonia (MPP) and 80 with nonrefractory MPP. All patients had fever $\left(38^{\circ} \mathrm{C}\right.$ or higher $)$. Written informed consent was obtained from all patients or the parents of all children. This study was conducted with the approval of the Institution Human Ethics Committee of Southern Medical University.

\section{Serologic tests}

Serum was obtained from all patients. Serum anti-MP antibody was detected by a PA assay using Serodia-MYCO II (Fuji Rebio Ltd., Tokyo, Japan) according to the manufacturer's instructions. Serum IgG- and IgM-specific anti-MP antibodies were determined using MP IgG and IgM ELISA kits (Savyon Diagnostics, Ashdod, Israel) following the manufacturer's instructions. Briefly, serum was diluted (1:200) and incubated on a plate coated with MP antigens. The optical density was detected by a TECAN-sunrise microplate reader and converted to an antibody value using a standard curve. Serum IgG- and IgM-specific anti-MP antibodies were also performed using the MP IgG and IgM CLIA kits (YHLO Biotech, Shenzhen, China) according to the manufacturer's instructions on a YHLO iFlash 3000 CLIA analyzer. Briefly, $50 \mu \mathrm{L}$ calibrators/serum samples and $50 \mu \mathrm{L}$ horseradish peroxidase enzyme-labeled triiodothyronine analog were added into the wells of the coated plate and incubated at $37^{\circ} \mathrm{C}$ for $45 \mathrm{~min}$. All unbound components were aspirated by the washer and washed 5 times using washing buffer. After an enzymatic reaction, the light emitted from the chemiluminescent reaction was measured by the chemiluminescence microplate reader and was proportional to the free triiodothyronine content in each well.

\section{Statistical analysis}

The statistical analyses were performed using SPSS 22.0 statistical software (IBM SPSS, Chicago, IL, USA). The kappa coefficient was calculated. A larger positive kappa value indicates a higher consistency between two methods. Kappa $\geq 0.75$ indicates good consistency, $0.75>$ kappa $\geq 0.4$ for medium consistency, and kappa $<0.4$ for poor consistency. ${ }^{12}$

\section{Results \\ Comparison of the three methods to detect the results of MP antibodies}

One hundred and eighty cases $(64.3 \%)$ were positive and 100 cases $(35.7 \%)$ were negative as measured by PA. For CLIA, 184 cases (65.7\%) were MP-IgM positive and 96 cases (34.3\%) were negative; 89 cases $(31.8 \%)$ were MP-IgG 
positive and 191 cases (68.2\%) were negative. For ELISA method, 196 cases were MP-IgM positive and 84 cases (30\%) were negative; MP-IgG was positive in 114 cases (40.7\%) and negative in 166 cases.

In view of the PA method being used for many years and identical to the clinics, the experimental data to PA method were based on the results for discussion. The results were divided into seven groups according to the MP antibody titer tested by PA method (<1:40, 1:40, 1:80, 1:160, 1:320, 1:640, $\geq 1: 1,280$, antibody titer $\geq 1: 80$ is positive). The comparison results of CLIA and ELISA detection of MP antibody were as follows: the positive rates of MP-IgM detected by CLIA were $22.8 \%$ (titer $<1: 40$ ) and $51.2 \%$ (titer=1:40), respectively. The positive rates of MP-IgM detected by ELISA were 33.3\% (titer $<1: 40$ ) and $53.5 \%$ (titer $=1: 40$ ), respectively.

In PA-positive group (titer $\geq 1: 80$ ), there was a decreasing trend of MP-IgM negative ratio measured by CLIA and ELISA. The negative ratios were $40 \%, 18 \%, 14.3 \%, 10 \%$, and $6.7 \%$ by CLIA and $43.3 \%, 14.3 \%, 5 \%$, and $6.7 \%$ by ELISA, respectively. However, the positive rate of MP-IgG showed an increasing trend: $13.3 \%, 26 \%, 42.9 \%, 75 \%$, and $91.1 \%$ by CLIA and $13.3 \%, 42 \%, 57.1 \%, 95 \%$, and $95.6 \%$ by ELISA, respectively. In the positive titer of 1:80-1:320, the total antibody $(\operatorname{IgM}+\operatorname{IgG})$ negative of MP accounted for a certain proportion (4\%-40\%; Table 1; Figure 1).

\section{The consistency analysis of CLIA and ELISA with PA}

Consistency check of CLIA and ELISA methods with PA in the antibody titer grouping was investigated. The results showed that the consistency coefficients of MP-IgM detected by CLIA and ELISA were 77\% (titer $<1: 40$ ), 49\% (titer=1:40), and $67 \%$ (titer $<1: 40$ ) and $47 \%$ (titer $=1: 40$ ), respectively, in PA-negative group. The consistent coefficients of MP-IgG detected by CLIA and ELISA were 100\% (titer $<1: 40$ ), $98 \%$ (titer $=1: 40$ ), and 95\% (titer $<1: 40)$ and 91\% (titer=1:40), respectively. The total percentages detected by CLIA and ELISA of MP total antibody (IgM+IgG) were $77 \%$ (titer $<1: 40$ ), $47 \%$ (titer $=1: 40$ ), and 65\% (titer $<1: 40$ ) and $47 \%$ (titer=1:40), respectively. In the PA-positive group, the consistent percentages of the two types of antibodies increased with raised MP antibody titers (Table 2; Figures 2 and 3).

\section{CLIA and ELISA assay MP antibody typing consistency analysis}

The concordance test showed that the negative consistencies of MP-IgM, MP-IgG, and MP total antibody (IgM+IgG)

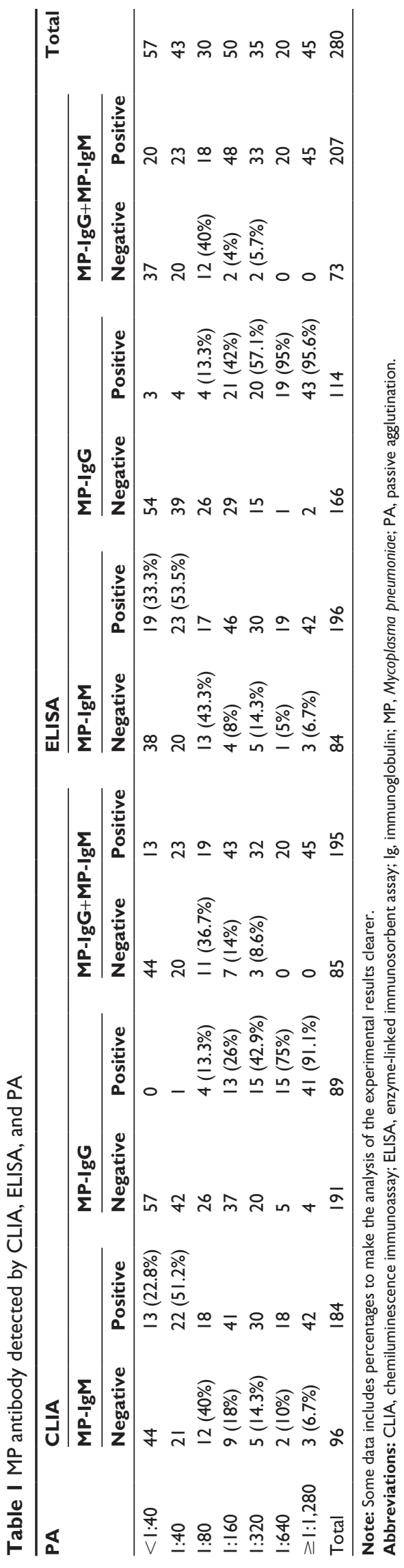


A

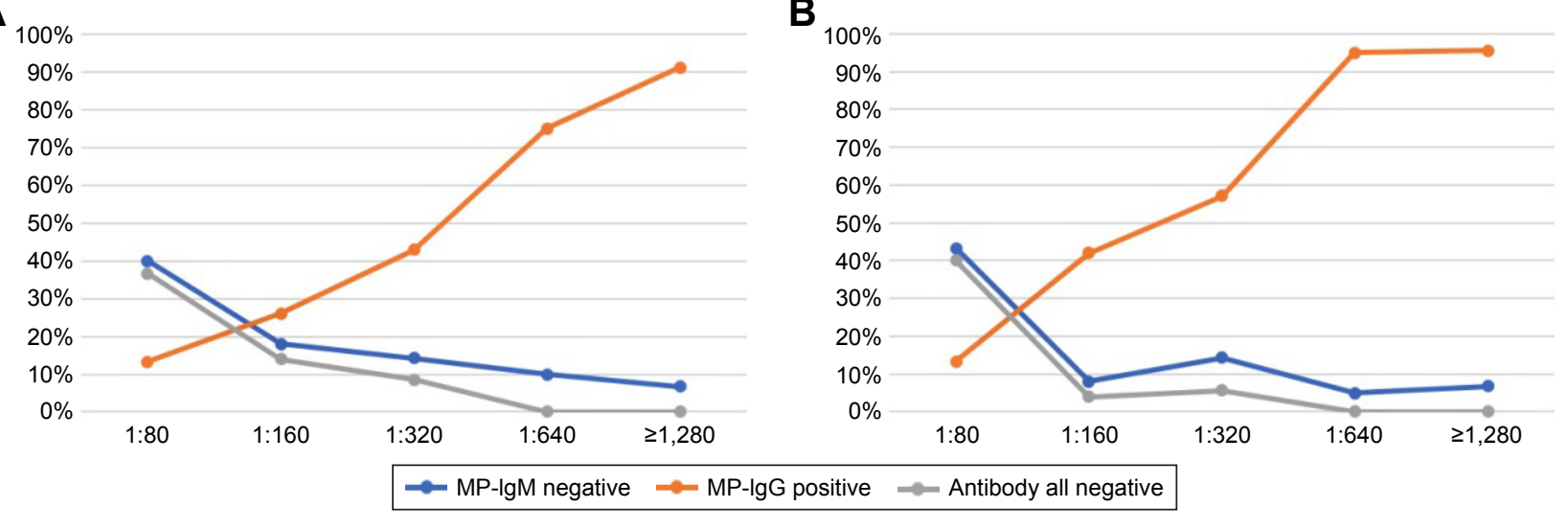

Figure I Antibody type and total antibody measured by CLIA (A) and ELISA (B) in PA-positive group.

Note: There was a decreasing trend of MP-IgM negative ratio while there was an increasing trend of MP-IgG positive ratio measured by CLIA and ELISA in PA-positive group (titer $\geq 1: 80$ ).

Abbreviations: CLIA, chemiluminescence immunoassay; ELISA, enzyme linked immunosorbent assay; Ig, immunoglobulin; MP, Mycoplasma pneumoniae; PA, passive agglutination.

groups were all higher than $92 \%$ and the positive consistencies of MP-IgM, MP-IgG, and MP total antibody (IgM+IgG) groups were all more than $75 \%$. The ratios of consistency of MP-IgM, MP-IgG, and MP total antibody (IgM+IgG) groups were all over $88 \%$, respectively. Kappa coefficients were $0.804,0.763$, and 0.806 in the MP-IgM, MP-IgG, and MP total antibody $(\operatorname{IgM}+\operatorname{IgG})$ groups (Table 3; Figure 4).

\section{Discussion}

In PA-negative group, CLIA and ELISA methods could detect a certain proportion of MP-IgM, indicating that the sensitivity of CLIA and ELISA was higher than that of PA. If the low MP-IgM concentration in patients can be detected, misdiagnosis rate could be dramatically decreased and the diagnosis and treatment efficiency can be improved. In the PA-positive group, the ratio of negative MP-IgM detected by CLIA and ELISA showed a decreasing trend, and the proportion of positive MP-IgG showed an increasing trend.
This result indicated that the higher the antibody titer of PA, the lower the negative rate of MP-IgM but the higher the positive rate of MP-IgG. Moreover, the percentage of MP total antibody $(\operatorname{IgM}+\operatorname{IgG})$ was overcast $(4 \%-40 \%)$. This result demonstrated that MP antibody detected by PA may contain IgM and IgG and other types of antibodies so that it has limitations on the clinical guidance. PA test kit does not specify the type of MP antibody in its instruction. Domestic and foreign PA commonly tests MP-IgM..${ }^{13-15}$ It is also reported that the PA method detects total antibody of MP or contains IgA antibodies. ${ }^{16,17}$ This study confirmed that the titer of MP antibody detected by the PA method (SERODIAMYCO II by Fuji Biosciences Co., Ltd.) included other types of antibodies except for IgM and IgG.

CLIA and ELISA methods were consistent with the PA method. Our results in Table 2 showed the increasing trend of CLIA and ELISA consistency coefficients with the raised MP antibody titers detected by PA (titers $\geq 1: 80$ ), which

Table 2 The consistency of CLIA and ELISA with PA

\begin{tabular}{|c|c|c|c|c|c|c|c|c|}
\hline \multirow[t]{2}{*}{ Consistency coefficient $\%$} & \multicolumn{7}{|l|}{ PA } & \multirow[t]{2}{*}{ Total } \\
\hline & $<\mathrm{I}: 40$ & $1: 40$ & $1: 80$ & $1: 160$ & $1: 320$ & $1: 640$ & $\geq \mathrm{I}: \mathbf{I}, \mathbf{2 8 0}$ & \\
\hline \multicolumn{9}{|l|}{ CLIA } \\
\hline MP-IgM & $77 \%$ & $49 \%$ & $60 \%$ & $82 \%$ & $86 \%$ & $90 \%$ & $93 \%$ & $76 \%$ \\
\hline MP-lgG & $100 \%$ & $98 \%$ & $13 \%$ & $26 \%$ & $43 \%$ & $75 \%$ & $91 \%$ & $67 \%$ \\
\hline$M P-\lg G+\lg M$ & $77 \%$ & $47 \%$ & $62 \%$ & $86 \%$ & $91 \%$ & $100 \%$ & $100 \%$ & $80 \%$ \\
\hline \multicolumn{9}{|l|}{ ELISA } \\
\hline MP-IgM & $67 \%$ & $47 \%$ & $57 \%$ & $92 \%$ & $86 \%$ & $95 \%$ & $93 \%$ & $76 \%$ \\
\hline MP-lgG & $95 \%$ & $91 \%$ & $13 \%$ & $42 \%$ & $57 \%$ & $95 \%$ & $96 \%$ & $71 \%$ \\
\hline MP-IgG+lgM & $65 \%$ & $47 \%$ & $60 \%$ & $96 \%$ & $94 \%$ & $100 \%$ & $100 \%$ & $79 \%$ \\
\hline
\end{tabular}

Abbreviations: CLIA, chemiluminescence immunoassay; ELISA, enzyme-linked immunosorbent assay; Ig, immunoglobulin; MP, Mycoplasma pneumoniae; PA, passive agglutination. 


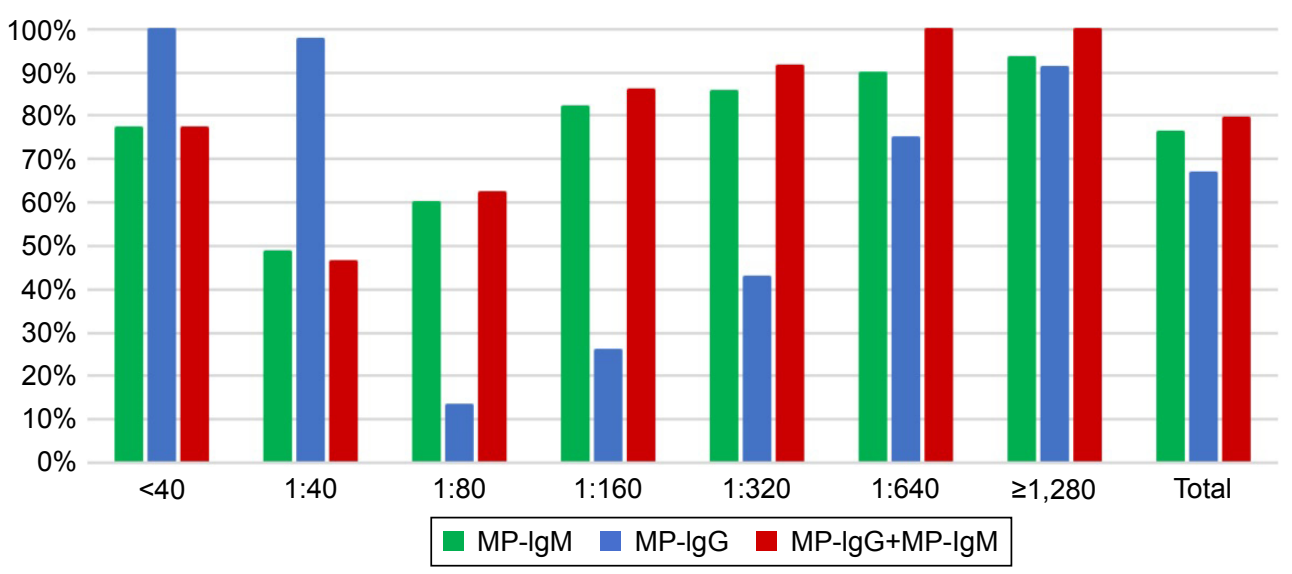

Figure 2 The consistency analysis of CLIA and PA.

Note: In the PA-positive group, the consistent percentages of the two types of antibodies increased with raised MP antibody titers.

Abbreviations: CLIA, chemiluminescence immunoassay; Ig, immunoglobulin; MP, Mycoplasma pneumoniae; PA, passive agglutination.

means that CLIA and ELISA have high consistency with PA. Compared with those of the PA method, the consistency coefficients $(76 \%$ and $76 \%$ ) of CLIA and ELISA for the detection of MP-IgM were higher than those of MP-IgG (67\% and 71\%). CLIA and ELISA had better agreement with the PA when ignoring antibody types ( $\operatorname{IgM}+\operatorname{IgG})$. These results are in good accordance with the results of Ouyang Fang. ${ }^{18}$ Their test results showed that the consistency of MP$\operatorname{IgG}(\mathrm{kappa}=0.702)$ was higher than MP-IgM (kappa=0.256). Results of the patients with MP-IgG and/or MP-IgM positive were identical to the PA result, with the consistency coefficient being higher (kappa=0.746). The differences could be caused by differences between different ELISA kits.

The consistency coefficients of MP-IgM, MP-IgG, and MP total antibody (IgM+IgG) detected by CLIA and ELISA were all higher than $75 \%$. Kappa coefficients were 0.804 ,
0.763 , and 0.806 , respectively. These results indicated that CLIA and ELISA kits were competent in clinical application for MP type detection.

MP infection can cause MPP. The incidence of MPP is slow and clinical manifestations are complicated and difficult to be recognized. If patients do not have timely diagnosis and treatment in its long course, other respiratory diseases and a variety of extrapulmonary complications may occur, which results in great threat to the growth and development of children. ${ }^{19}$ Therefore, early diagnosis and treatment is particularly important. The significance of laboratory tests for the diagnosis of MP infection has become increasingly apparent. However, the reported positive rates and infection rates vary widely. The traditional culture methods of mycoplasma, so-called "gold standard", need high requirements, and the positive rate is very low because of the

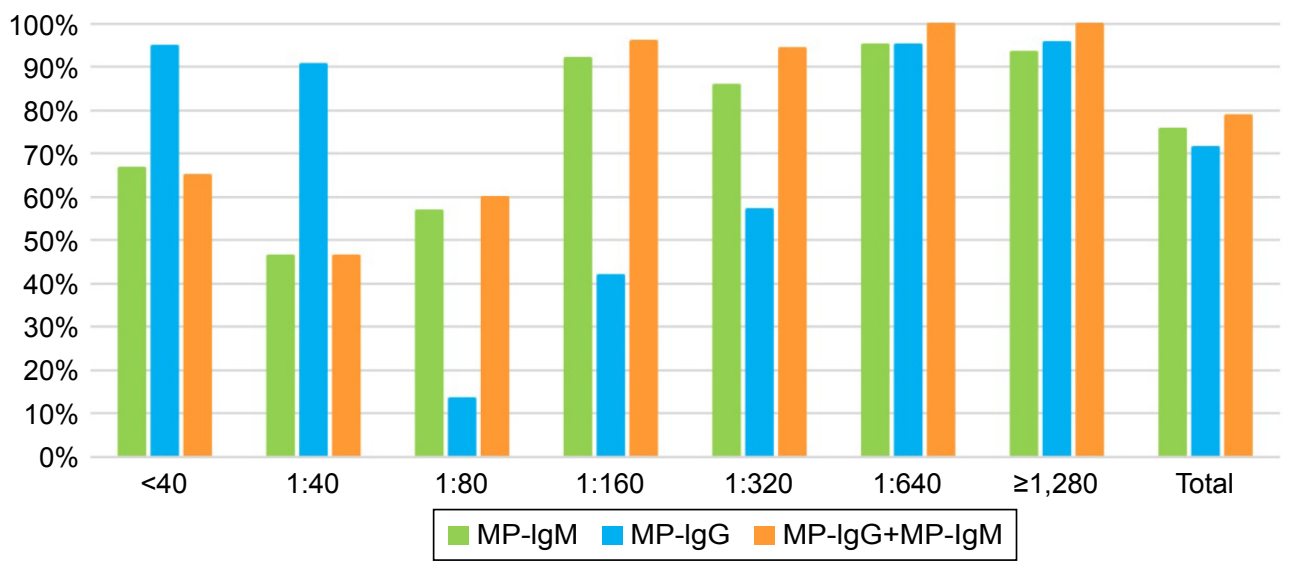

Figure 3 The consistency analysis of ELISA and PA.

Notes: In the PA-positive group, the consistent percentages of the two types of antibodies increased with raised MP antibody titers.

Abbreviations: ELISA, enzyme-linked immunosorbent assay; Ig, immunoglobulin; MP, Mycoplasma pneumoniae; PA, passive agglutination. 
Table 3 The consistency of CLIA and ELISA in MP antibody typing

\begin{tabular}{|c|c|c|c|}
\hline & MP-IgM & MP-IgG & MP-IgG+IgM \\
\hline Consistency coefficient (\%) & 91.4 & 88.9 & 92.1 \\
\hline Negative consistency (\%) & 92.9 & 98.2 & 93.2 \\
\hline Positive consistency (\%) & 90.8 & 75.4 & 91.8 \\
\hline Kappa & 0.804 & 0.763 & 0.806 \\
\hline
\end{tabular}

Abbreviations: CLIA, chemiluminescence immunoassay; ELISA, enzyme-linked immunosorbent assay; Ig, immunoglobulin; MP, Mycoplasma pneumoniae.

factors such as the sampling process, culture conditions, and season, ${ }^{20,21}$ which take a long time and greatly limit its clinical application. Therefore, it was not a common inspection item in clinics. Molecular biology methods such as realtime PCR and Western blot are other detection methods in the diagnosis of MP infection, but it cannot meet the needs of rapid clinical diagnosis because it is time-consuming and complicated. ${ }^{22}$

ELISA method can identify antibody type and its positive predictive value is high. Nevertheless, due to incubation, washing, and other operations, batch testing methods are used in the clinical laboratory. Usually, clinical laboratory tests only one batch of ELISA a day and need to wait for $>24 \mathrm{~h}$ to get the results. Thus, ELISA does not apply to a single test and rapid test. The sensitivity of the ELISA (SeroMP IgG/ IgM ELISA kit) in this study is higher than that of the PA. However, Chen et $\mathrm{al}^{23}$ concluded that the sensitivity of the ELISA (AUMON, Lübeck, Germany) was lower than that of the PA, probably due to the different quality of numerous ELISA kits and the use of laboratory operations. The fact that CLIA provide antibody titers in a faster, simpler, and more objective manner than ELISA results in limited use of ELISA. PA method does not require that the plate be washed and the operation is simple, so in recent years more clinics use PA to detect MP-IgM. However, this study showed that MP detected by PA has complex composition, including IgM, IgG, or even other types of antibodies. Patients may be misdiagnosed by results of PA. Mycoplasma antigen composition is complex. Different MP antigen epitopes respond separately on antibodies causing different cross-reactions. This is one of the reasons why different kits may have different results. Mycoplasma pneumonia can evade the body's immune defenses and avoid the macrophage phagocytosis through the variation of surface antigen, leading to clinically "refractory" MPP. ${ }^{24}$

\section{Conclusion}

The detection sensitivity of CLIA and ELISA is higher than that of PA. When the concentration of MP-IgM in patients is low, CLIA and ELISA can detect the antibody, reducing the rate of misdiagnosis. The higher the antibody titer of PA method, the higher the positive consistency coefficient of CLIA and ELISA with PA. CLIA and ELISA detection of MP typing antibody has high consistency with PA. Compared with the existing manual and semiautomatic detection methods (PA and ELISA), automated chemiluminescence (CLIA) much more meets the need of clinical test and can be considered as priority in clinical application because of its specificity, sensitivity, and accuracy.

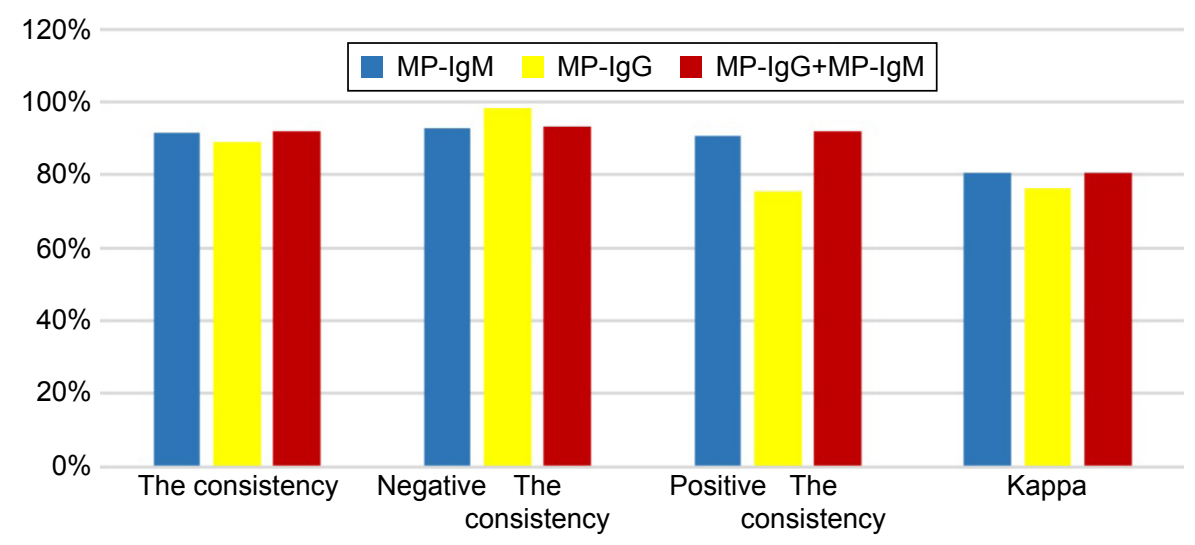

Figure 4 The consistency analysis of CLIA and ELISA in typing MP antibody.

Note: The results showed consistency between CLIA and ELISA.

Abbreviations: CLIA, chemiluminescence immunoassay; ELISA, enzyme-linked immunosorbent assay; Ig, immunoglobulin; MP, Mycoplasma pneumoniae. 


\section{Disclosure}

The authors report no conflicts of interest in this work.

\section{References}

1. Meyer Sauteur PM, van Rossum AM, Vink C. Mycoplasma pneumoniae in children: carriage, pathogenesis, and antibiotic resistance. Curr Opin Infect Dis. 2014;27(3):220-227.

2. Gotoh K, Nishimura N, Ohshima Y, et al. Detection of Mycoplasma pneumoniae by loop-mediated isothermal amplification (LAMP) assay and serology in pediatric community-acquired pneumonia. J Infect Chemother. 2012;18(5):662-667.

3. Zhou Z, Li X, Chen X, et al. Comparison of P1 and 16S rRNA genes for detection of Mycoplasma pneumoniae by nested PCR in adults in Zhejiang, China. J Infect Dev Ctries. 2015;9(3):244-253.

4. Chen Z, Yan Y, Wan Y, et al. Epidemiology of community acquired Mycoplasma pneumoniae respiratory tract infections among hospitalized Chinese children, including relationships with meteorological factors. Hippokratia. 2013;17(1):20-26.

5. Waites KB, Balish MF, Atkinson TP. New insights into the pathogenesis and detection of Mycoplasma pneumoniae infections. Future Microbiol. 2008;3(6):635-648.

6. Cherry JD, Quanquin NM. Mycoplasma and ureplasma infections. In: Cherry JD, Harrison GJ, Kaplan SL, et al, editors. Feigin and Cherry's Textbook of Pediatric Infectious Diseases. 7th ed. Philadelphia, PA: Elsevier Saunders; 2014:2668-2687.

7. Atkinson TP, Balish MF, Waites KB. Epidemiology, clinical manifestations, pathogenesis and laboratory detection of Mycoplasma pneumoniae infections. FEMS Microbiol Rev. 2008;32(6):956-973.

8. Daxboeck F, Krause R, Weinsch C. Laboratory diagnosis of Mycoplasma pneumoniae infection. Clin Microbiol Infect. 2003;9(4):263-273.

9. Medjo B, Atanaskovic-Markovic M, Radic S, Nikolic D, Lukac M, Djukic S. Mycoplasma pneumoniae as a causative agent of communityacquired pneumonia in children: clinical features and laboratory diagnosis. Ital J Pediatr. 2014;40(1):104.

10. Wang L, Feng Z, Zhao M, et al. A comparison study between GeXPbased multiplex-PCR and serology assay for Mycoplasma pneumoniae detection in children with community acquired pneumonia. BMC Infect Dis. 2017;17(1):518.

11. Lu Z, Zhong N. Medicine. 7th ed. Beijing: People's Medical Publishing House; 2008:11-13.
12. Xia B, Wu J. Application of kappa consistency test in laboratory medicine. Chinese J Lab Med. 2006;29(1):83-84.

13. Lee S-C, Youn Y-S, Rhim J-W, et al. Early Serologic Diagnosis of Mycoplasma pneumoniae Pneumonia: an Observational Study on Changes in Titers of Specific-IgM Antibodies and Cold Agglutinins. Medicine. 2016;95(19):e3605.

14. Waites KB, Lanier Thacker W, Talkington DF. The value of culture and serology for detection of Mycoplasma pneumoniae infections in the clinical laboratory in the age of molecular diagnostics. Clin Microbiol Newsl. 2017;23:123-129.

15. Yamazaki T, Narita M, Sasaki N, Kenri T, Arakawa Y, Sasaki T. Comparison of PCR for sputum samples obtained by induced cough and serological tests for diagnosis of Mycoplasma pneumoniae infection in children. Clin Vaccine Immunol. 2006;13(6):708-710.

16. Chertsak Dhiraputra MD. Serological study of Mycoplasma pneumoniae infections. J Med Assoc Thai. 2004;87(8):935-938.

17. Beersma MFC, Dirven K, van Dam AP, Templeton KE, Claas ECJ, Goossens H. Evaluation of 12 commercial tests and the complement fixation test for Mycoplasma pneumoniae-specific immunoglobulin G (IgG) and IgM antibodies, with PCR used as the "gold standard." J Clin Microbiol. 2005;43(5):2277-2285.

18. Xu D, Li S, Chen Z, Du L. Detection of Mycoplasma pneumoniae in different respiratory specimens. Eur J Pediatr. 2011;170(7):851-858.

19. Youn Y-S, Lee K-Y. Mycoplasma pneumoniae pneumonia in children. Korean J Pediatr. 2012;55(2):42-47.

20. Ma LD, Chen B, Dong Y, et al. Rapid mycoplasma culture for the early diagnosis of Mycoplasma pneumoniae infection. J Clin Lab Anal. 2010;24(4):224-229.

21. Zhang L, Zong Z-Y, Liu Y-B, Ye H, Lv X-J. PCR versus serology for diagnosing Mycoplasma pneumoniae infection: a systematic review \& meta-analysis. Indian J Med Res. 2011;134:270-280.

22. Daxboeck F, Krause R, Wenisch C. Laboratory diagnosis of Mycoplasma pneumoniae infection. Clin Microbiol Infect. 2003;9(4):263-273.

23. Chen Q, Li Y, Tao C, et al. [The significance of the differences between the detection methods of Mycoplasma pneumoniae antibody]. J Clin Exp Med. 2015;14(22):1850-1853. Chinese.

24. Wang M, Wang Y, Yan Y, et al. Clinical and laboratory profiles of refractory Mycoplasma pneumoniae pneumonia in children. Int $J$ Infect Dis. 2014;29:18-23.
Therapeutics and Clinical Risk Management

\section{Publish your work in this journal}

Therapeutics and Clinical Risk Management is an international, peerreviewed journal of clinical therapeutics and risk management, focusing on concise rapid reporting of clinical studies in all therapeutic areas, outcomes, safety, and programs for the effective, safe, and sustained use of medicines. This journal is indexed on PubMed Central, CAS,

\section{Dovepress}

EMBase, Scopus and the Elsevier Bibliographic databases. The manuscript management system is completely online and includes a very quick and fair peer-review system, which is all easy to use. Visit http://www.dovepress.com/testimonials.php to read real quotes from published authors. 